\title{
CREACIÓN Y MEDIATIZACIÓN: ORLANDO RINCÓN Y PARQUESOFT: ECOSISTEMA TECNOLÓGICO
}

ENTREVISTA A ORLANDO RINCÓN

PRESIDENTE EJECUTIVO DE PARQUESOFT

Por

Paula Marcela Trujillo

Comunicadora Social 


\section{Resumen:}

Entrevista a Orlando Rincón, Presidente Ejecutivo de ParqueSoft, conglomerado informático en el que converge la ciencia, la tecnología, el arte y el entretenimiento. Las factorías, las empresas del Parque, desarrollan productos de diferente naturaleza y carácter. Comprender los modos de interacción, los avances tecnológicos, los factores que rodean la creación tecnológica y la trayectoria de este pionero del software y el emprendimiento empresarial en Colombia, son algunos de los motivos del presente material.

ParqueSoft es una empresa de arte digital, ciencia y tecnología informática, nacida en Cali en 1999, con sede también en otras ciudades de Colombia, que cuenta con más de 200 aplicaciones para empresas de salud, gubernamentales, agroindustria, educación y entretenimiento. Creación de software, consultoría tecnológica, prestación de servicios de outsourcing y producción de cine, video, animación y micromovies, son algunos de los productos que ofrecen, siendo este cluster uno de los conglomerados informáticos más grandes en América Latina.

Orlando Rincón, su presidente ejecutivo y uno de los fundadores de ParqueSoft, nos concedió esta entrevista en diciembre de 2010 como parte del Trabajo de Grado de la Maestría en Sociología Creación multimedial y mediatización: aproximación a creadores, diseñadores y productores de animación, portales de internet, webs TV, blogs y videojuegos en Cali.

A sus 48 años, Orlando Rincón, Ingeniero de Sistemas de la Universidad EAFIT de Medellín, confiesa que antes de dedicarse al área tecnológica, estudió seis semestres de Antropología en la Universidad de Antioquia. De estrato dos pasó a estrato seis, dice entre risas, pues cuando se formó no sabía nada de la vida ni del mundo y hoy cree saber un poco más, dado el nivel intelectual y profesional forjado. Gestar un complejo industrial como este parque tecnológico, denominado, por él, como un ecosistema, no sólo significa cambios en los modos de hacer empresa y generar empleo, sino también en el abordaje técnico-científico de interesantes maneras de creación. La entrevista que presentamos consta así de dos líneas temáticas, que se entremezclan; por un lado, se encuentra ParqueSoft como empresa y, por otro, Orlando Rincón, como pionero del desarrollo de tecnologías de software en Colombia.

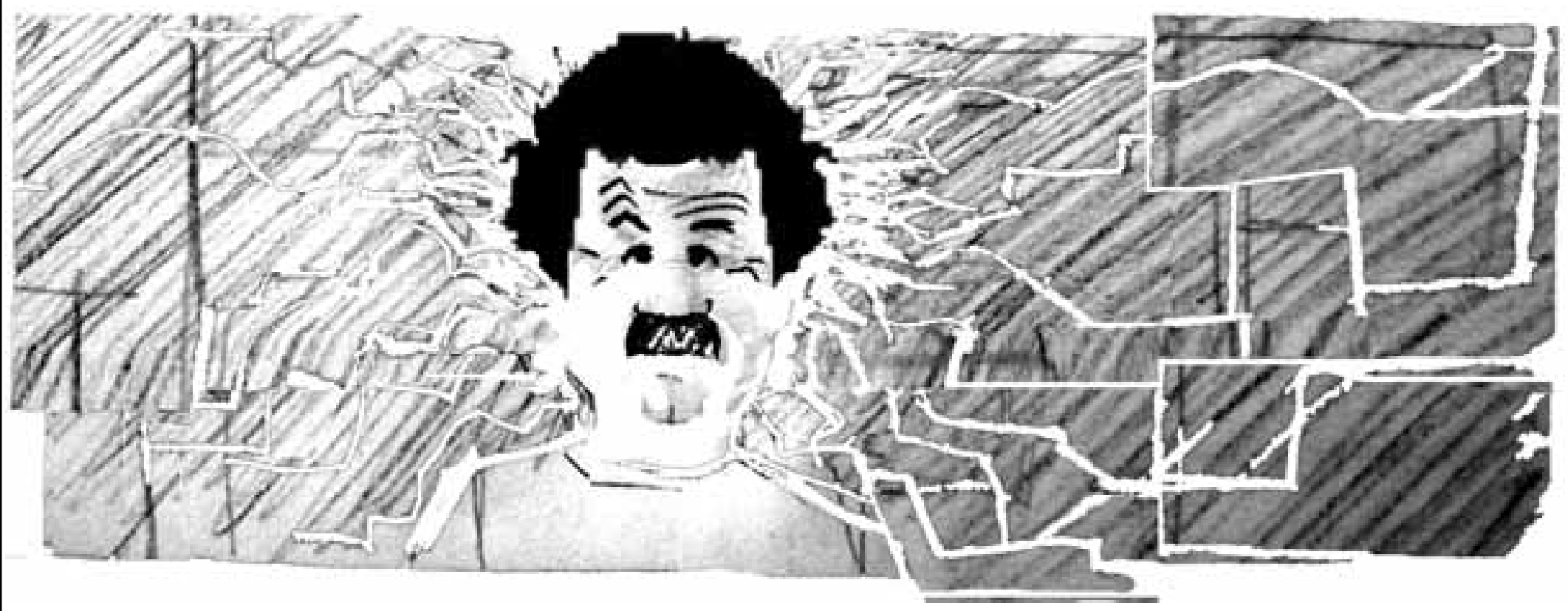




\section{¿Cómo se presenta la empresa, cómo se ha presentado? ¿Ha ido cambiando su perfil?}

Nosotros creamos en 1999 ParqueSoft y hemos sido coherentes desde el primer día. El objetivo era crear un ecosistema en donde se juntara gente de ciencias, de las ingenierías y de las artes, alrededor de las tecnologías de la información y la comunicación. Desde el primer día vislumbramos tres áreas muy importantes de trabajo: el nuevo paradigma que había al principio del año 2000 con el nacimiento de la internet, el mantenimiento de los sistemas de información requeridos para las grandes empresas, para el gobierno y para las actividades de la sociedad y el advenimiento de todo un mundo de entretenimiento y uso digital de las tecnologías de la información, como una plataforma de reemplazo de todas las tecnologías análogas. Eso hacía que se visualizara una masificación muy grande con la computación móvil y, por lo tanto, la demanda de contenido iba a ser también muy grande, porque iba a ser personalizada; se presentaba así un mercado inmenso.

\section{¿Cómo opera ParqueSoft?}

ParqueSoft es un ecosistema de emprendimiento, este tema lo conocemos muy bien, desde hace muchísimos años, antes que se implementara en universidades y en otros espacios. Se ha tratado de especular mucho sobre métodos, desde las universidades y desde algunas instituciones, pero nosotros siempre hablamos de las teorías biológicas, por eso no hablamos de un centro de desarrollo empresarial, hablamos de un ecosistema. Los emprendedores en ParqueSoft no crean empresas por capital, se crean empresas por emprendedores y esa es la diferencia. No estamos ni con el mercado de valores, ni con accionistas, actuamos como y con emprendedores. Un emprendedor llega a ParqueSoft, es seleccionado de una manera natural y comienza su proceso. Uno puede identificar unas etapas que son las naturales de nacimiento, supervivencia, sostenibilidad y luego de sustentabilidad. La desaparición en el caso nuestro, como en la naturaleza, es de transformación no es una muerte. Pero esas etapas no tienen un tiempo definido, no tienen un método específico porque dependen de muchas cosas; dependen de la base sociocultural del emprendedor; si el emprendedor viene de estratos populares su proceso y desarrollo es muy diferente, a si viene de clase media o alta. Segundo, también depende de dónde proviene, si llega de una escuela de arte, de una universidad pública, de una universidad privada o si sencillamente es una persona emprendedora natural sin educación. La otra variable importante es su capacidad sicológica de conectarse, eso es lo que más procura el ecosistema ParqueSoft, pero no lo impone, se tiene que dar de una manera natural; entre más se demore el emprendedor en encontrar el valor de energizarse y conectarse, pues más tiempo se va a demorar en llegar a la etapa de sustentabilidad. 
La otra variable importante es su capacidad de conectarse con el mercado, eso depende de la habilidad, hay emprendedores muy buenos pero que no logran un desarrollo importante porque no tienen la habilidad de conectarse con el mercado y eso tampoco depende de una metodología, descansa sobre una cantidad de variables: de la innovación, de la tecnología, de su equipo de trabajo, de su posición, de sus relaciones y esencialmente del producto y servicio que está intentando desarrollar. Nosotros entendemos que son muchas variables y no presionamos, porque creemos que todo tiene su desarrollo natural, es por eso que hablamos de un ecosistema que muestra caminos. Hay unidades empresariales que no han pasado de estar integradas por dos personas. Como en todos los ecosistemas hay especies pequeñas, medianas, grandes, hay especies dominantes, dominadas, unas que tienen más potencial para desarrollarse, unas que tienen más habilidades que otras, otras que se conectan más rápido y se expanden y ocupan territorio, es la ley natural.

\section{¿Antecedentes suyos, actividades, profesiones, roles en los que se movían sus padres influyeron en su decisión de estudiar ingeniería de sistemas y crear una empresa? ¿Hay una relación entre formación familiar y lo que hace hoy?}

Mi madre y mi padre pertenecían a un hogar muy humilde. Ninguo de los dos tenía edcuación, pero él era muy emprendedor. Siempre tuvo trabajo como independiente y tuvo maquinaria pesada. Mi madre era muy sensible y mi padre era una persona con una inteligencia genética. De ellos obviamente heredé mi estructura biológica, pero hubo una cosa muy importante: nosotros no tuvimos religión. Creo que eso fue muy importante para que en nuestra familia existiera un ecosistema de ciencia. Había una postura abierta frente a contenidos, frente a la ciencia, frente a la tecnología, especialmente en la época en la que crecí, en la que la religión era determinante. Eso influyó muchísimo un pensamiento totalmente abierto.

\section{¿ Hay gustos de infancia qué pudieron verse reflejados en su trabajo?}

Mucho gusto por el cine, por el contenido audiovisual, por la literatura y la ciencia. También siempre he tenido mucha relación con la naturaleza y con proyectos sociales muy grandes. Siempre he tenido un contacto muy fuerte con el campo. Casi todas las actividades que hacemos colaterales están relacionadas con llevar tecnologías de la información a las escuelas y a las comunidades; realizamos diversas dinámicas de responsabilidad social desde las TICS. 


\section{¿Cómo es su recorrido empresarial?}

Desde muchacho participé de muchas actividades. Cuando estaba en la universidad comencé vendiendo unas computadoras caseras que salieron en 1982. Mi primer trabajo fue hacer juegos para esas máquinas, jueguitos en resolución mínima, entretenimiento. Comencé a hacer los juegos populares de calle: los cinco huecos, el dominó. Los vendía a los mismos clientes que compraban los computadores. Luego participé en muchas creaciones audiovisuales. De hecho, en el contenido audiovisual de ParqueSoft, que es bastante extenso, alrededor de 100 audiovisuales con fines institucionales, en muchos he participado creativamente. El contenido ha sido totalmente educativo o institucional, no comercial. Desde el primer día estuve en compañías de internet, de videojuegos, de diseño gráfico y cine.

Primero, en Parquesoft nos dedicamos a producir contenidos creativos para plataformas educativas. Luego vino Antorcha, la primera empresa que comenzó a trabajar con contenido multimedial en ParqueSoft. Los contenidos de los parques temáticos también los he creado.

\section{Entonces, su relación con la creación...}

Viene desde los $80^{\prime}$ s con la creación de software, pero antes había creado el periódico del colegio, un pasquín. Hay 14 ParqueSoft y 3 parques temáticos. Nosotros hicimos Prolisa, el parque que quedaba en Centenario, era una nave espacial donde la gente jugaba y que murió porque al centro comercial no le llegaron los visitantes que nos habían prometido y se quebró. Luego hicimos el parque informático en Popayán, tenía como 50 contenidos, con mecatrónica y mucha animación.

Luego del Parque Informático de Popayán hicimos Astrolita, una creación muy linda con electrónica y software para los niños. Posteriormente, hicimos Imaginera, el cual cerró el gobernador Abadía, allí habían 80 contenidos. Proponer las ideas, el trabajo creativo, la creación, me gusta, pero lo que más placer me produce es cuando lo ves hecho realidad. Hoy hay una relación más alta de la tecnología con la creación. Creo que falta preguntarse por el futuro a mediano y corto plazo, qué, cómo, de eso puede salir una variable muy interesante, se necesitarían unos cuadros de reemplazo muy acelerados para mantener la industria y para acrecentarla. En el mundo del software el tipo llega, comienza a programar y a los dos años ya no quiere hacerlo más, quiere volverse gerente. Esa es una de las cosas que está afectando esta industria, cada vez los hacedores de la cosas hacen push, dejan de hacer y no llegan tantos para seguir haciendo. 


\section{¿Cuáles son sus rutinas, hábitos mediáticos y estéticos?}

Veo televisión entre las diez de la noche y las tres de la mañana, todos los días. Tengo cinco criterios de observación: el primero son los canales científicos (Discovery Channel, History Channel y Natgeo). Segundo los canales de noticias e información: CNN, NTN24 y algo de Al Jazeera, BBC de Londres, TV5 y Telesur. Tercero, en orden de relevancia, es el cine: Cinemax, especialmente. Cuarto: canales culturales, el Canal Gourmet y los canales de culinaria. Con la música me relaciono menos que con la televisión, mucha salsa, música clásica y Jazz. En radio sólo escucho los programas deportivos del medio día. Con la prensa impresa mi relación es fuerte. Tengo suscripción a siete revistas que leo juiciosamente y a dos periódicos que leo todos los días (El País y Portafolio). Leo Semana, Dinero, América Economía, la revista de Mac y una revista especializada nuestra que se llama Wiki Manager y American Science.

\section{¿Cómo es su relación con los libros?}

Muy buena. Una mezcla de todo, principalmente literatura. No leo nada de literatura de administración y ya no leo libros de ciencias porque ya todo lo leo en revistas o en artículos de la internet. Todos los libros que leo son fundamentalmente sobre literatura y cultura. Consumo bastante teatro también, voy cada ocho días. Teatro local y el internacional que llega.

\section{¿Y el cine y los videojuegos?}

Con Simón y Bruno vemos mucha comedia y películas de acción. Me interesan también contenidos especiales: cine independiente. En videojuegos solamente participo en los laboratorios de videojuegos que hay en el parque, pero lo hago por ayudar. Me mantengo todo el tiempo conectado, a través del celular el portátil y cuando no los tengo a la mano, cafés internet en los aeropuertos, en donde sea, pero todo el tiempo conectado.

\section{¿De todas estas prácticas, rutinas, cuál ha sido la que más ha marcado su labor de creación?}

El cine es una retroalimentación. Te voy a decir una cosa, te vas a sorprender, casi la mayoría de los conceptos de ParqueSoft los he creado yo. Cuando hacemos los brainstories, casi la mayoría de ideas han salido de mi cabeza y el 95\% han sido influidas por mi relación con el cine. Desde el punto de vista sicológico, cognitivo, desde el punto de vista de conocimientos específicos y de imaginarios audiovisuales. Obviamente la literatura también, la ciencia ficción, he sido muy influenciado por ese imaginario futurista. 
¿Si fuéramos a hacer una reflexión, a partir de toda su experiencia, en cuánto a evoluciones tecnológicas, en términos de lenguaje y evoluciones estéticas, qué podríamos decir? ¿Del 82 para acá cómo se ha evolucionado?

Hay cinco variables que mueven toda esta evolución: la primera es el aumento progresivo de la definición que cambia todo y posibilita pasar de una matriz de 80x24 a una de millones por millones, en tan solo 20 años. Las primeras cosas que hice eran en una resolución de 80x24. Hoy la resolución que tiene mi computador en píxeles es de 2000 x 2000 y pasamos de 2600 a 4 millones de puntos. El segundo factor es la operación, pasamos de 600 mil instrucciones por segundo a teras, o sea millones de millones de instrucciones por segundo. La tercera cosa es el sonido, pasamos de unas calidades de sonido análogo al sonido digital, que da unas posibilidades impresionantes; la cuarta es la tecnología software, pasamos de unas herramientas costosas, pesadas, difíciles de administrar, a unas libres, baratas, livianas, poderosísimas y con millones de habilidades que han potenciado a un montón de creadores en el mundo. La quinta variable importante es que internet ha permitido mirar y comparar un subconjunto de creaciones muy chiquiticas, que era a lo que uno tenía acceso en las bibliotecas. Hoy todo lo que crea la raza humana está en internet.

\section{¿Qué entiende por estética?}

La estética es, bajo la lectura que nosotros hacemos, la forma como ciertos grupos sociales hacen esa relación entre lo que les produce tranquilidad, placer y gusto, frente a lo que otros producen. De ahí que sea tan complejo este tema porque existen estéticas económicas, culturales y biológicas. Es un concepto influenciado por tantos factores que, obviamente los fenómenos de globalización, han producido un cierto nivel de propuestas de estéticas de las que la industria local no se puede alejar.

La manga es toda una estética de la animación y hay otras que proveen una estética no solo audiovisual sino que imponen un fenómeno global como Los Simpsons. Existen también estéticas microlocales, locales, regionales y nacionales; estéticas internacionales y globales y, por otro lado, existen estéticas étnicas y de clases sociales. Es muy interesante porque siento que allí hay una tremenda oportunidad para la industria, porque sólo se ha concentrado en cuatro estéticas de consumo global, pero hay ejemplos, inclusive en Colombia donde muchas propuestas creativas se han basado en estéticas regionales, locales y han sido muy exitosas, como la de Martín de Francisco y la que produce la Barra del América Barón Rojo que tiene una estética muy específica. El colectivo fanático escarlata, ha creado su propia estética local, dirigida a esa barra y adaptada al paradigma de Cali y en especial a los actores populares, mezcla de indígena y afro, una propuesta muy carnavalesca. 
El cine es el arte del siglo XX. ¿Cuál cree que pueda ser el arte del siglo XXI?

La inteligencia artificial, los sistemas expertos y el nuevo poder de los computadores que han bajado de tamaño y que permiten hoy inteligencias extrasensoriales, como la realidad aumentada, la biogenética y las experiencias sensoriales. Todo tiene que ver con el cuerpo humano que va a cambiar. El cine sigue siendo un arte que predomina por la relación cultural, pero cada vez las unidades de entretenimiento personal, individual, están dominando más, se están imponiendo.

\section{¿Relaciona eso más con ciencia o con arte? ¿Cree que el placer, el entretenimiento y la ciencia están estrechamente relacionados?}

El entretenimiento hoy es la base que está generando toda la demanda. Todavía hay unos testarudos que persisten en crear contenidos de arte y eso está bien a nivel de propuestas de creación y de cómo el ser humano puede producir unas lecturas, como existió en el pasado con Hollywood. Uno veía películas de unos tres o cuatro locos que creaban una cosa completamente diferente, pero con esta globalización creo que cada vez va a ser menos. En mi opinión, todavía, el siglo XXI no se ha definido, estamos en sus albores. No quiero imaginar en el 2050 de qué estaremos hablando. De sensaciones debajo de la piel y tú ya no verás con los ojos.

\section{Howard Rheingold dice que el estímulo va a llegar directamente a la retina, sin pantalla...}

Vamos a tener tanto dominio de la neurociencia que vamos a tener, de pronto, otra forma de entender. Hasta ahora todo nuestro feedback ha sido a través de los ojos, los oídos, los cinco sentidos externos, porque tenemos estas interfaces para tocar el mundo. Pero en el momento en que toquemos directamente el cerebro, para qué se necesitan las manos, los ojos, la lengua o los oídos, si puedes ir directamente al centro de procesamiento y joh sorpresa! de pronto descubrimos que somos capaces de procesar otros sentidos que hoy en día no conocemos. Ya se está hablando de cuarta dimensión, de universos paralelos, de física cuántica. Si hay algo que presiona los límites de la ciencia es el consumo masivo y el principal elemento de consumo masivo es el entretenimiento. No se producen tecnologías para trabajar, no se producen tecnologías para vivir, se producen tecnologías esencialmente para gozar y hacen que la gente consuma; lo que ha producido el desborde de la ciencia es el entretenimiento. ¿Cuál es el medio? Hay un solo medio, cuando uno habla de multimedia está hablando de todo, del celular, del Facebook, Google es multimedia. 


\section{¿Qué sería estética multimedial? ¿Cuáles pueden ser aquellos criterios estéticos en relación con este lenguaje digital?}

Depende de lo que quieres hacer, existen muchas personas que hacen creaciones individuales que por accidente o no, las ponen en un medio como: Youtube, Facebook y un montón de gente las ve y terminan siendo eso: cosas de consumo masivo que no fueron diseñadas para eso. Eso es lo bonito de esas plataformas que están destruyendo todos esos estereotipos de marketing, de creación. Si te sientas en Youtube o te pones a ver contenidos en Facebook, no las fotos, sino lo que la gente hace, te garantizo que en menos de una hora has visto más de mil estéticas. Realmente ese es un tema muy complejo. Si estás en la industria, obviamente, debes seguir unos parámetros estéticos, pero si no estás y eres un actor paralelo a la industria, que no participa de ella, pero termina haciéndola, un actor indirecto, no existe ninguna limitación estética. Muchas creaciones se vuelven globalmente exitosas y terminan imponiéndose. Hay un par de chicos que puedes ver en internet que doblan canciones y les meten animación. Comenzaron jugando, son un par de pelados de 17 años y los vieron sus compañeros del colegio, hoy los ve todo el mundo, han creado una estética de mamar gallo que está siendo imitada en todo el mundo. Eso no lo hicieron para venderlo, es freeweb. Esas explosiones creativas terminan volviéndose productos y servicios globales y casi que diariamente están cambiando las estéticas, se proponen nuevas o se reinventan.

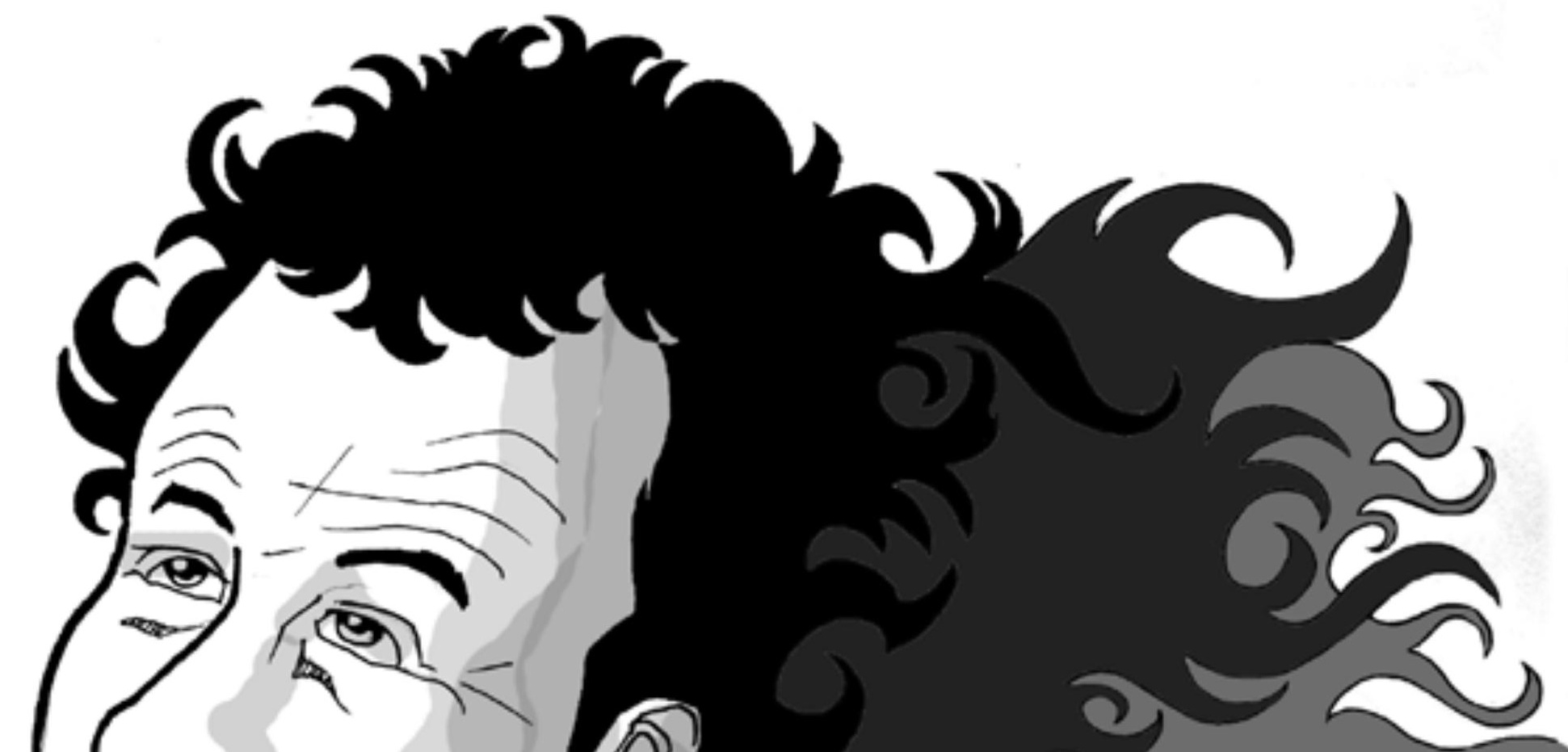




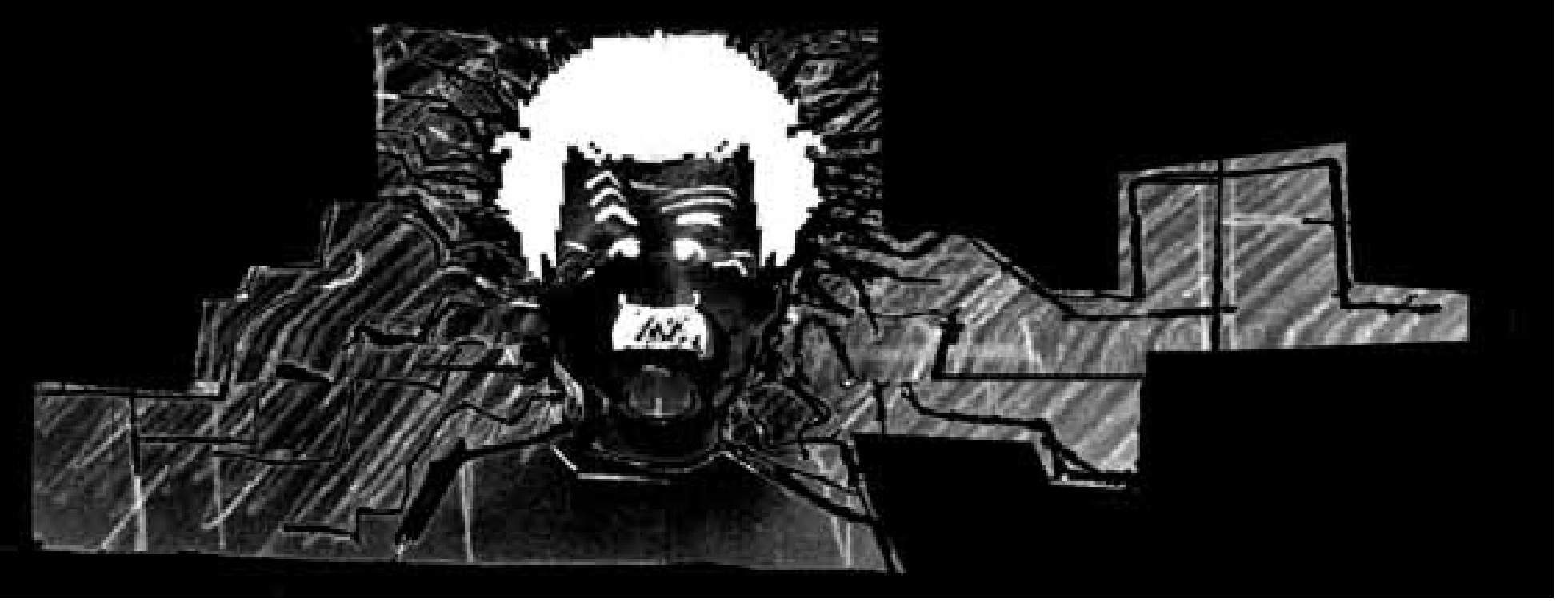

\section{Si nos concentramos en diseño creativo y creación de software, ¿cómo es la creación, el desarrollo creativo?}

Alrededor de las tecnologías de la información, las grandes creaciones resultan de la innovación, pero no todas resultan innovadoras y no necesariamente son a través de un producto o mediante tecnologías. Pueden ser servicios que se ofrecen sobre tecnologías ya existentes y que permiten grandes revoluciones sociales o de mercado. Era muy popular que todas las cosas salieran de los laboratorios de la IBM, de los laboratorios de General Electric, de la Siemens. Hoy salen millones de ideas de parques, de casas, de iglesias, de escuelas, de individuos solos, conectados a internet. Antes era la figura de un laboratorio cerrado y ahora todo es ubicuo, todo es etéreo, todo el mundo está innovando. Hoy en día las empresas ya no son sólo laboratorios de diseño, ahora son estudios. En muchos de los estudios el creativo construye, a diferencia de las agencias de publicidad en las que el creativo solo cuenta las ideas y otros las producen. En nuestra industria los creativos también son hacedores, son programadores, animadores o productores. Son work groups, células de desarrollo, muy parecidas a la guerra de guerrillas, grupos pequeños, donde hay un líder y se mueven rápidamente y todos hacen de todo. El único rol que no se comparte es el del programador porque la computación gráfica es muy exigente. Los artistas no pueden programar porque no tienen los conocimientos, el programador sí conoce todas las herramientas de diseño y los efectos y es capaz de hacerlo todo. Cuando me refiero a realidad aumentada y a estas cosas, a medios interactivos, éste debe ser el centro. La animación no es interactiva. Si pasas por un bombillo se prende y la animación se mueve y el piso se abre. Pero si vos tenés un website y decís escríbeme un banner aquí y te pone el banner, ya no necesitas ni diseñadores ni ingenieros. 

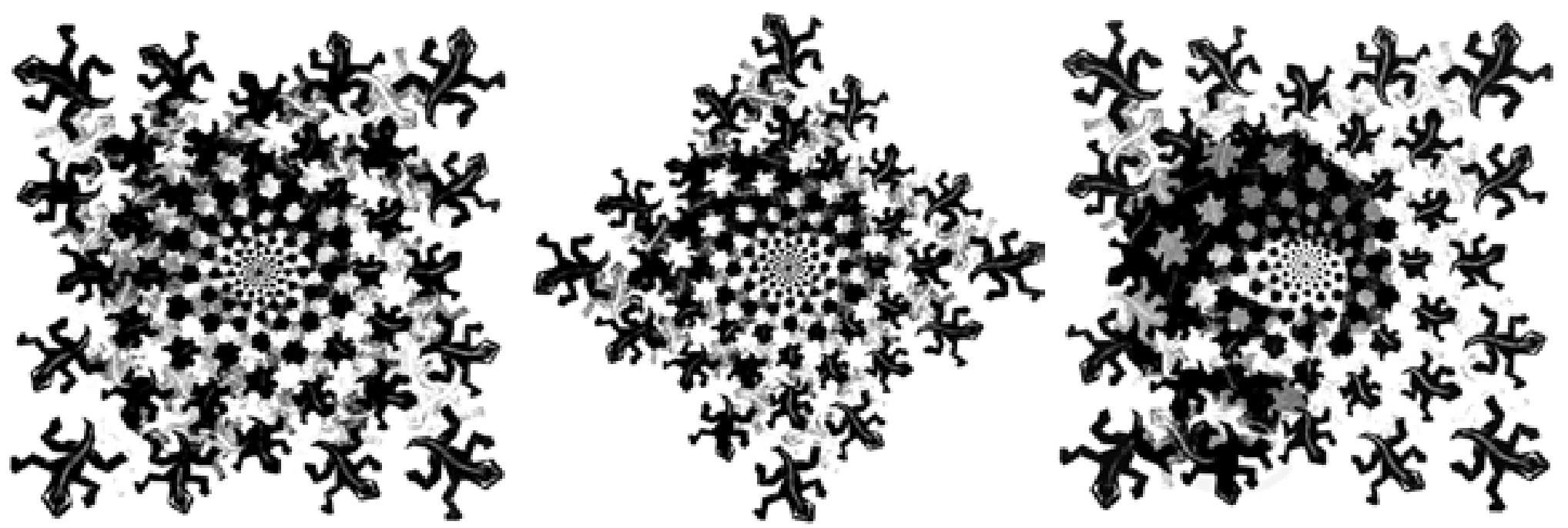

Digamos entonces que el arte electrónico, el arte multimedial, el arte digital...

Son tecnologías del presente. Internet ha permitido que mucha gente desde su casa con presupuestos chiquititos puedan pensar cosas y hacer experimentos e interfaces que antes no eran posibles. ¿Qué estará ocurriendo en cientos de cuartos de casas en este momento?, ¿dónde debe haber chicos o señores pensando en otra manera de comunicarse, en destruir la televisión, por ejemplo? Un mundo sin televisión es posible. ¿Quién se imaginaba la vida con Facebook hace 5 años?. La tecnología es la que le da el peso a la idea.

¿Relaciona creación multimedial con entretenimiento

y con arte; también con industria cultural?

Y con la educación: "educainment" y

"artentertainment”.

La educación con entretenimiento, pero es dominante

el entretenimiento.

Inevitablemente si estamos hablando de entretenimiento,

hablamos de un negocio, de un mercado, de una industria...

Mucho de lo que consume la gente hoy en día es free. Los bananeros crean contenidos y no cobran y se entretienen y entretienen.

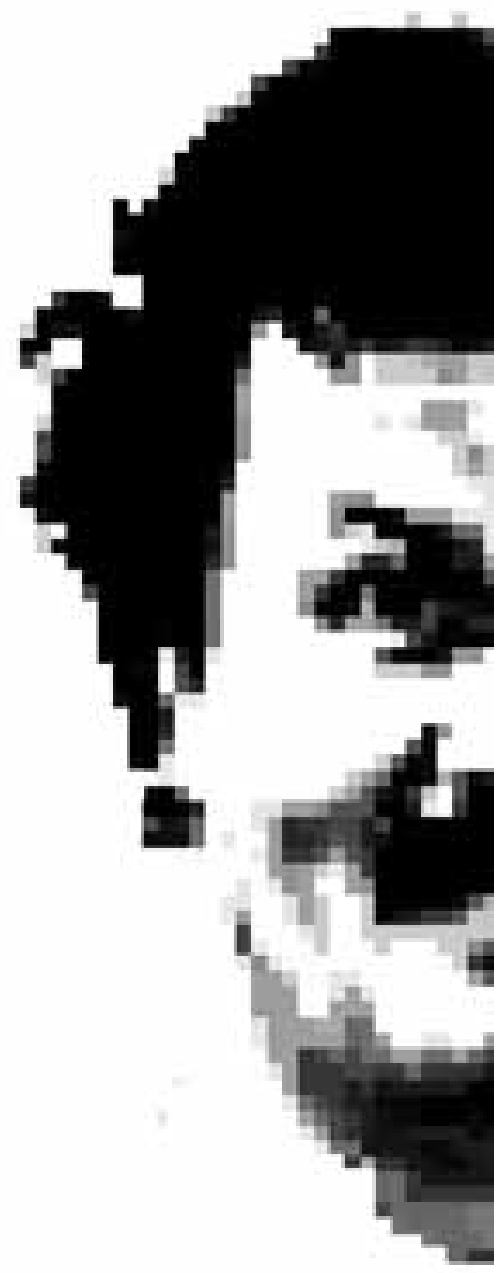




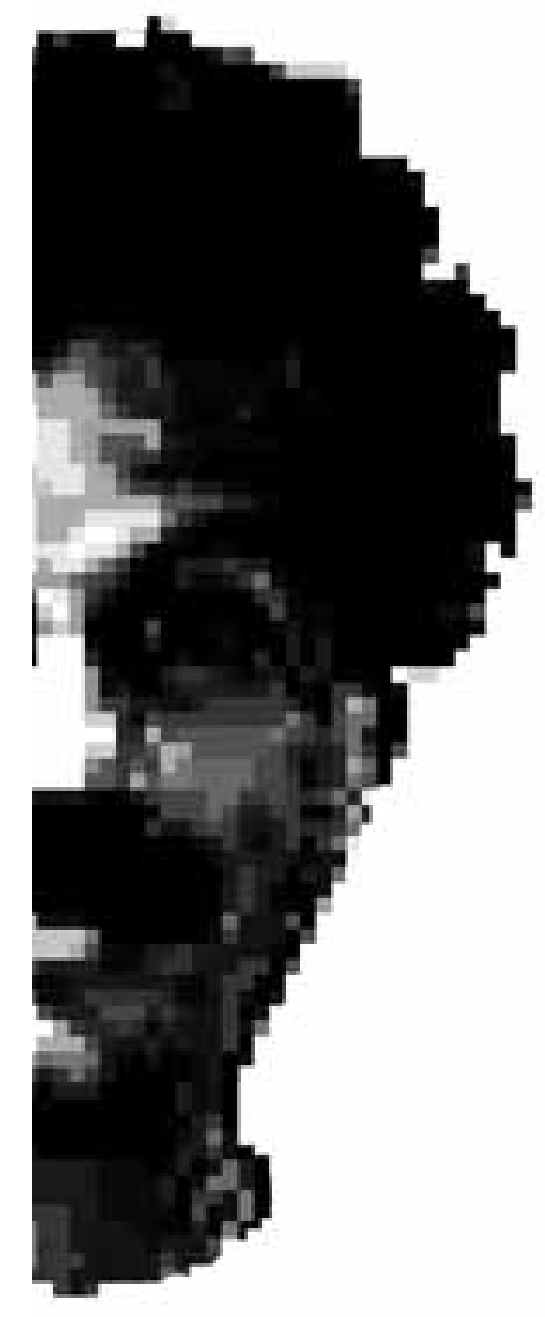

\section{Pero de todas maneras estarían las industrias creativas, industrias culturales, ahí sí hay un lucro...}

Sí, de todas maneras pese a que hay mucho contenido libre, todavía hay mucho por el cual se debe pagar y sigue siendo un excelente negocio. La industria de juegos tiene sus capas, nosotros tenemos cuatro compañías de videojuegos para celulares, hay unas que hacen juegos para consolas, para el $X$-box, para el Playstation, otras diseñan juegos para PC que no corren en Play o Nintendo y otrass como nosotros están haciendo juegos para ipad, iphone y celulares que no funcionan en esos otros dispositivos. Esta industria está muy golpeada también porque tú creas muchas cosas, pero no son creaciones tuyas. Todos quieren inventarse la rueda, por eso es que esta industria no progresa porque mientras tú creas toda tu línea pasan años.

La industria del cine todavía sigue siendo muy vigente, tiene un lógica totalmente diferente. La industria de los micromovies no tiene nada que ver con la industria del cine, porque los micromovies los hace una persona con un celular, con una cámara web o con una cámara de esas baratas, toman una situación y no dura más de un minuto, entonces no tiene luces, ni técnico, ni logística, ni producción, ni almuerzos. Es otro mundo, un micromovie hace millones. En Colombia la industria del micromovie no existe porque hacer una buena película en menos de un minuto es difícil pero es rentable porque un micromovie lo descargan dos millones de personas a 50 centavos de dólar. Cien dólares vendidos en menos de una semana. Cuando ves una película como Perro come perro juntar un millón de dólares no es posible, ni porque la vean en 50 festivales alrededor del mundo, en cambio, un micromovie recoge un millón de dólares y al tipo le costó dos tardes. 